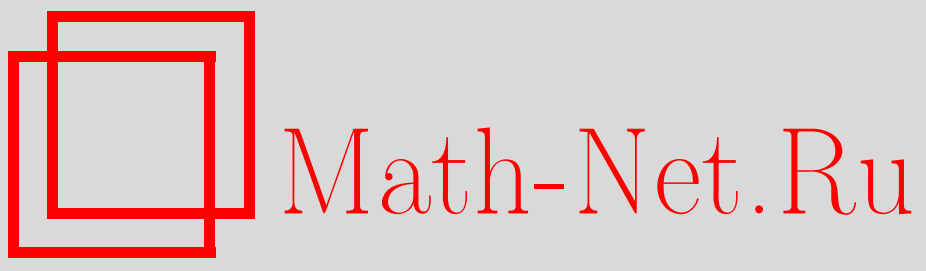

С. В. Савченко, Об изменении спектральных свойств матрицы при возмущении достаточно низкого ранга, Функи. анализ и его прил., 2004, том 38, выпуск $1,85-88$

DOI: https://doi.org/10.4213/faa100

Использование Общероссийского математического портала MathNet.Ru подразумевает, что вы прочитали и согласны с пользовательским соглашением

http://www. mathnet.ru/rus/agreement

Параметры загрузки:

IP : 3.85 .5 .30

26 апреля 2023 г., 15:39:51

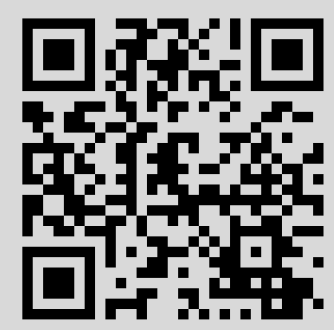


УДК $512.643+517.983$

\title{
Об изменении спектральных свойств матрицы при возмущении достаточно низкого ранга
}

\author{
(C) 2004. C. B. САВчЕНKO
}

Пусть $A$ и $B$ - любые две квадратные матрицы одинакового порядка. Существует большое количество статей и даже книг (см., например, [1-3]), в которых строится теория возмущений для собственных значений матрицы $A+\epsilon B$, не входящих в спектр матрицы $A$. Однако если геометрическая кратность $k$ собственного значения $\lambda$ матрицы $A$ больше ранга $r$ матрицы $B$, то $\lambda$ должно остаться в спектре новой матрицы $A+B$ (здесь и везде в дальнейшем мы не налагаем никаких ограничений на норму матрицы $B$ ). Как при переходе от $A$ к $A+B$ изменится часть жордановой формы, соответствующая $\lambda$ ? Как ни странно, в литературе отсутствует ответ на этот естественный вопрос. В нашей работе мы покажем, что при типичном возмущении $B$ ранга $r$ у $\lambda$ пропадают $r$ самых больших жордановых клеток, а порядки остальных не изменяются.

Для суммы произведений соответствующих координат любых двух векторов $\xi$ и $\eta$ введем обозначение $(\xi, \eta)$. Тогда любой оператор $B$ ранга $r$ можно представить в виде $B=\sum_{i=1}^{r}\left(\cdot, \xi_{i}\right) \eta_{i}$, где $\left\{\xi_{i}\right\}_{i=1}^{r}$ и $\left\{\eta_{i}\right\}_{i=1}^{r}$ - две системы линейно независимых векторов. Обозначим через $A(z)$ матрицу скалярных резольвент, $i j$-элемент которой равен $\left((z E-A)^{-1} \eta_{j}, \xi_{i}\right)$, где $i, j=1, \ldots, r$. Рассмотрим ограничение оператора $B(z E-A)^{-1}$ на линейную оболочку $\left\langle\eta_{1}, \ldots, \eta_{r}\right\rangle$ векторов $\eta_{1}, \ldots, \eta_{r}$. Очевидно, что его матрица в базисе $\left\{\eta_{i}\right\}_{i=1}^{r}$ совпадает с $A(z)$. Так как прямая сумма корневых подпространств оператора $B(z E-A)^{-1}$, соответствующих его ненулевым собственным значениям, содержится в $\left\langle\eta_{1}, \ldots, \eta_{r}\right\rangle$, то справедливо следующее тождество:

$$
\frac{\operatorname{det}(z E-A-B)}{\operatorname{det}(z E-A)}=\operatorname{det}\left(E-B(z E-A)^{-1}\right)=\operatorname{det}(E-A(z)) .
$$

Пусть $d-$ количество всех жордановых клеток в жордановой форме матрицы $A$ и $n_{1}, \ldots, n_{d}$ - их порядки (мы считаем, что клетка с номером $p$ соответствует собственному значению $\lambda_{p}$ ). Возьмем любой жорданов базис $\left\{\xi_{n}^{(p)}\right\}_{n=1}^{n_{p}} \begin{gathered}d \\ p=1\end{gathered}$ матрицы $A$ и дуальный к нему жорданов базис $\left\{\eta_{n}^{(p)}\right\}_{n=1}^{n_{p}} \stackrel{d}{p=1}$ транспонированной матрицы $A^{\top}$. Здесь $\left(A-\lambda_{p} E\right) \xi_{n}^{(p)}=\xi_{n-1}^{(p)}$ и $\left(A^{\top}-\lambda_{p} E\right) \eta_{n}^{(p)}=\eta_{n-1}^{(p)}$ при $n=1, \ldots, n_{p}$ (мы считаем, что $\left.\xi_{0}^{(p)}=\eta_{0}^{(p)}=0\right)$. Дуальность означает, что $\left(\xi_{n^{\prime}}^{\left(p^{\prime}\right)}, \eta_{n}^{(p)}\right)=\delta_{n^{\prime}, n_{p}+1-n} \delta_{p^{\prime}, p}$, где $\delta-$ символ Кронекера. Нетрудно показать справедливость представления

$$
\left((z E-A)^{-1} \eta_{j}, \xi_{i}\right)=\sum_{p=1}^{d} \sum_{n=1}^{n_{p}}\left(\eta_{n}^{(p)}, \eta_{j}\right) \sum_{m=1}^{n_{p}-n+1}\left(z-\lambda_{p}\right)^{-\left(n_{p}-n+2-m\right)}\left(\xi_{m}^{(p)}, \xi_{i}\right)
$$

Обозначим через $\Xi_{n}^{(p)}$ матрицу, $i m$-элемент которой равен $\left(\xi_{m}^{(p)}, \xi_{i}\right)$, где $i=$ $1, \ldots, r$ и $m=1, \ldots, n_{p}-n+1$. Пусть $\Psi_{n}^{(p)}$ есть $r \times\left(n_{p}-n+1\right)$-матрица, каждый элемент $j$-й строки которой совпадает с $\left(\eta_{n}^{(p)}, \eta_{j}\right)$, и $\Delta_{n}^{(p)}(z)$ - диагональ- 
ная матрица с элементами $\left(z-\lambda_{p}\right)^{-n_{p}+n-1}, \ldots,\left(z-\lambda_{p}\right)^{-1}$ на диагонали. Тогда $i j$-элемент произведения $\Xi_{n}^{(p)} \Delta_{n}^{(p)}(z) \Psi_{n}^{(p) \top}$ совпадает с выражением в (2), следующим после знака суммы $\sum_{n=1}^{n_{p}}$. Обозначим через $\Xi$ и $\Psi$ матрицы, полученные последовательным расположением друг за другом всех матриц $\Xi_{n}^{(p)}$ и $\Psi_{n}^{(p)}$ coответственно, не заключенных, как обычно, в скобки (матрица с индексом $n+1$ следует за матрицей с индексом $n$, серия матриц с номером $p+1$ следует за серией матриц с номером $p)$. Пусть $\Delta(z)=\Delta_{1}^{(1)}(z) \oplus \cdots \oplus \Delta_{n_{d}}^{(d)}(z)-$ прямая сумма всех диагональных матриц $\Delta_{n}^{(p)}(z)$, упорядоченных указанным выше способом. Тогда $A(z)=\Xi \Delta(z) \Psi^{\top}$. В дальнейшем мы будем считать, что $\lambda_{1}=\cdots=\lambda_{k}=\lambda$ и $n_{1} \geqslant \cdots \geqslant n_{h}>n_{h+1}=\cdots=n_{r}=\cdots=n_{g}>n_{g+1} \geqslant \cdots \geqslant n_{k}$. Применяя формулу Бине-Коши (см. формулу (16) гл. I из [4]) к произведению матриц $\Xi \Delta(z)$ и $\Psi^{\top}$, получим, что порядок полюса функции $\operatorname{det} A(z)$ в точке $z=\lambda$ не больше, чем $n_{1}+\cdots+n_{r}$. Кроме того, коэффициент при $(z-\lambda)^{-\left(n_{1}+\cdots+n_{r}\right)}$ в разложении в ряд Лорана функции $\operatorname{det} A(z)$ совпадает с суммой

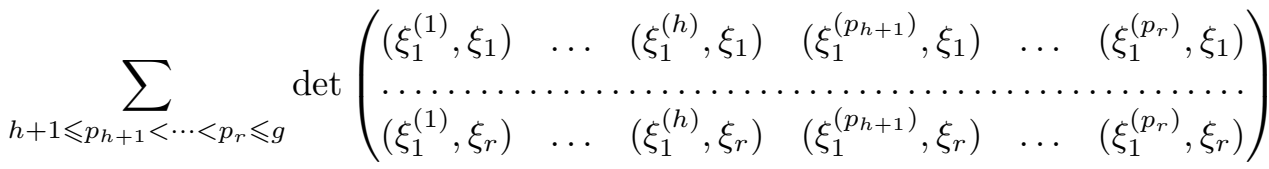

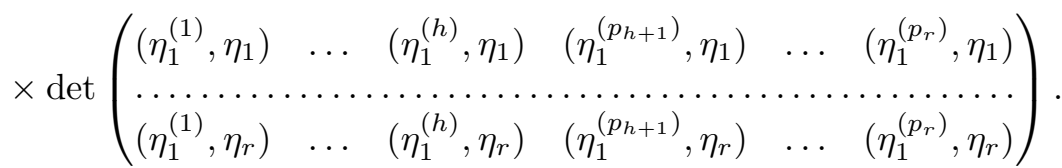

Нетрудно показать, что те же самые утверждения справедливы и для функции $\operatorname{det}(E-A(z))$. Обозначим через $n_{A}(\lambda)$ алгебраическую кратность $\lambda$ как собственного значения матрицы $A$. Очевидно, что $n_{A}(\lambda)=n_{1}+\cdots+n_{k}$. В силу тождества (1) справедливо неравенство $n_{A+B}(\lambda) \geqslant n_{A}(\lambda)-n_{1}-\cdots-n_{r}$, причем равенство в этом неравенстве имеет место тогда и только тогда, когда сумма (3) не равна нулю. Понятно, что это условие является типичным (т. е. справедливым для почти всех наборов векторов $\xi_{1}, \ldots, \xi_{r}$ и $\left.\eta_{1}, \ldots, \eta_{r}\right)$.

Теорема 1. Пусть $A-$ произвольная квадратная матрица, и пусть $B=$ $\sum_{i=1}^{r}\left(\cdot, \xi_{i}\right) \eta_{i}$ - оператор ранга $r$. Рассмотрим любое собственное значение $\lambda$ матрищы $A$ и упорядочим порядки его жсордановых клеток по невозрастанию, $n_{1} \geqslant \cdots \geqslant n_{k}$. Пусть $k \geqslant r$. Предположсим, что имеет место равенство

$$
n_{A+B}(\lambda)=n_{A}(\lambda)-n_{1}-\cdots-n_{r}
$$

Тогда $n_{r+1}, \ldots, n_{k}$ являются порядками жсордановых клеток матриць $A+B$, соответствующих собственному значению $\lambda$.

ДокАЗАтЕльство. Случай $k=r$ очевиден. Предположим, что $k>r$. Если сумма (3) не равна нулю, то не равен нулю хотя бы один из левых определителей в ней. Без ограничения общности можно считать, что

$$
\operatorname{det}\left(\begin{array}{ccc}
\left(\xi_{1}^{(1)}, \xi_{1}\right) & \ldots & \left(\xi_{1}^{(r)}, \xi_{1}\right) \\
\ldots \ldots \ldots \ldots & \ldots \ldots & \ldots \ldots \\
\left(\xi_{1}^{(1)}, \xi_{r}\right) & \ldots & \left(\xi_{1}^{(r)}, \xi_{r}\right)
\end{array}\right) \neq 0
$$


Пусть числа $\alpha_{n, 1}^{(p)}, \ldots, \alpha_{n, r}^{(p)}$ определяются рекуррентным образом по числам $\alpha_{1,1}^{(p)}, \ldots, \alpha_{1, r}^{(p)}, \ldots, \alpha_{n-1,1}^{(p)}, \ldots, \alpha_{n-1, r}^{(p)}$ как единственное решение системы уравнений

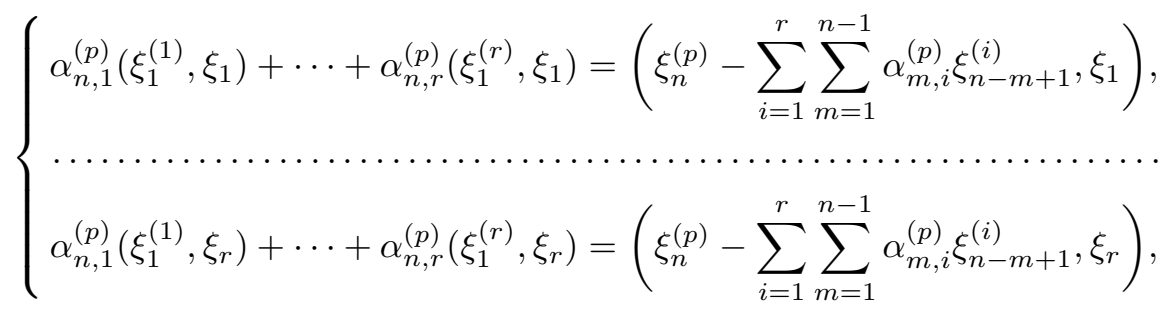

где $r+1 \leqslant p \leqslant k$ и $1 \leqslant n \leqslant n_{p}$ (при $n=1$ мы считаем, что все суммы $\sum_{m=1}^{n-1}$ равны нулю). Тогда вектор $\xi_{n}^{(p) \prime}=\xi_{n}^{(p)}-\sum_{i=1}^{r} \sum_{m=1}^{n} \alpha_{m, i}^{(p)} \xi_{n-m+1}^{(i)}$ ортогонален вектоpaм $\xi_{1}, \ldots, \xi_{r}$. Кроме того, $(A-\lambda E) \xi_{n}^{(p) \prime}=\xi_{n-1}^{(p) \prime}$. Поэтому $(A+B-\lambda E) \xi_{n}^{(p) \prime}=\xi_{n-1}^{(p) \prime}$ при $n=1, \ldots, n_{p}$ и $p=r+1, \ldots, k$ (здесь $\left.\xi_{0}^{(p) \prime}=0\right)$. В силу (4) число векторов в построенных цепочках равно размерности корневого подпространства $L_{A+B}(\lambda)$ матрицы $A+B$ для $\lambda$. Поэтому они образуют базис и, следовательно, в силу своей формы, жорданов базис в $L_{A+B}(\lambda)$. Теорема доказана.

Из (1) и (3) следует, что если справедливо равенство (4), то $n_{A+\epsilon B}(\lambda)=$ $n_{A}(\lambda)-n_{1}-\cdots-n_{r}$ при любом $\epsilon \neq 0$. В силу доказательства теоремы 1 имеем $B L_{A+B}(\lambda)=0$. Поэтому корневое подпространство $L_{A+\epsilon B}(\lambda)$ не зависит от $\epsilon \neq 0$ и совпадает с $L_{A+B}(\lambda)$. Обычно в теории возмущений используют матрицу $\mathbf{B}, p p^{\prime}$-элемент которой равен $\left(B \xi_{1}^{\left(p^{\prime}\right)}, \eta_{1}^{(p)}\right)$, где $p, p^{\prime}=1, \ldots, k$ (см. [3]). Нетрудно показать, что произведение двух определителей в сумме (3) равно главному минору порядка $r$ матрицы $\mathbf{B}$, соответствующему множеству индексов $\left\{1, \ldots, h, p_{h+1}, \ldots, p_{r}\right\}$. Предположим, что эта сумма не равна нулю и отличны от нуля все главные миноры порядка $q_{s} \leqslant h$ матрицы В в условии (1.6) теоремы 1 из [3]. Кроме того, пусть все ненулевые корни уравнения (1.9) различны. Тогда простая модификация теоремы Лидского, основанная на ее доказательстве, позволяет утверждать, что при разрушении каждой жордановой клетки порядка $n_{p}$, где $p=1, \ldots, r$, образуются $n_{p}$ простых собственных значений матрицы $A+\epsilon B$, которые с точностью до $o\left(\epsilon^{1 / n_{p}}\right)$ расположены в вершинах правильного $n_{p}$-угольника, вписанного в окружность радиуса порядка $\epsilon^{1 / n_{p}} \mathrm{c}$ центром в точке $\lambda$. В силу тождества (1) собственный вектор, соответствующий каждому такому собственному значению $\lambda(\epsilon)$, может быть представлен в виде $(A-\lambda(\epsilon) E)^{-1}\left(\alpha_{1} \eta_{1}+\cdots+\alpha_{r} \eta_{r}\right)$, где коэффициенты $\alpha_{i}$ являются координатами решения уравнения $\epsilon A(\lambda(\epsilon)) \boldsymbol{\alpha}=\boldsymbol{\alpha}$.

ПримЕчАнИЕ. В январе 2002 г. результаты о типичном изменении спектральных свойств при возмущении ранга $r$ были сообщены (без доказательства) автором Ж. Mopo (J. Moro) и Ф. Допико (F. Dopico) из Мадридского университета. Им также был отослан препринт, в котором содержится полное решение проблемы для случая $r=1$. Недавно они проинформировали автора, что ими подготовлена статья "Low rank perturbation of Jordan structure" для представления в SIAM Journal on Matrix Analysis and its Applications, в которой утверждение теоремы 1 настоящей работы доказывается в чисто матричных терминах, без использования резольвентной техники. 


\title{
ЛитеРАТУРА
}

1. Kato T. Perturbation Theory for Linear Operators. Springer-Verlag, Berlin, 1980. 2. Baumgartel $H$. Analytic Perturbation Theory for Matrices and Operators. Birkhäuser, Basel, 1985. 3. Лидский В. Б. Ж. вычисл. матем. и матем. физ., 6, № 1, 52-60 (1966). 4. Гантмахер Ф. Р. Теория матриц. М., Наука, 1967.

Институт теоретической физики им. Л. Д. Ландау РАН

Поступило в редакцию e-mail: sav@icp.ac.ru 3 октября 2002 г.

\section{УДК 517.57}

\section{Экстремальные тригонометрические и степенные многочлены нескольких переменных}

\author{
(c) 2004. Л. А. САхнович
}

1. Рассмотрим множество $\sigma_{P}$ степенных неотрицательных многочленов от нескольких переменных. Обозначим через $Q_{P}$ класс многочленов из $\sigma_{P}$, которые могут быть представлены в виде суммы квадратов. В классической работе Д. Гильберта [1] показано, что $Q_{P}$ не совпадает с $\sigma_{P}$. В настоящей заметке мы попытались выработать общий подход к исследованию экстремальных элементов выпуклых множеств $Q_{P}$. Статья базируется на следующей методике:

1) мы исследуем неотрицательные тригонометрические многочлены и затем с помощью преобразования Кальдерона переходим к степенным многочленам;

2) в статье дан способ построения опорных гиперплоскостей выпуклых множеств $Q_{P}$; этот факт играет важную роль в нашем подходе.

Обозначим через $\sigma\left(N_{1}, N_{2}, N_{3}\right)$ множество тригонометрических многочленов

$$
f(\alpha, \beta, \gamma)=\sum_{|k| \leqslant N_{1}} \sum_{|\ell| \leqslant N_{2}} \sum_{|m| \leqslant N_{3}} q(k, \ell, m) e^{i(k \alpha+\ell \beta+m \gamma)},
$$

удовлетворяющих условию $f(\alpha, \beta, \gamma) \geqslant 0(\alpha=\bar{\alpha}, \beta=\bar{\beta}, \gamma=\bar{\gamma})$. Обозначим через $Q\left(N_{1}, N_{2}, N_{3}\right)$ семейство тригонометрических многочленов класса $\sigma\left(N_{1}, N_{2}, N_{3}\right)$, допускающих представление

где

$$
f(\alpha, \beta, \gamma)=\sum_{j=1}^{r}\left|F_{j}(\alpha, \beta, \gamma)\right|^{2},
$$

$$
F_{j}(\alpha, \beta, \gamma)=\sum_{0 \leqslant k \leqslant N_{1}} \sum_{0 \leqslant \ell \leqslant N_{2}} \sum_{0 \leqslant m \leqslant N_{3}} q_{j}(k, \ell, m) e^{i(k \alpha+\ell \beta+m \gamma)} .
$$

2. Ясно, что множество $Q\left(N_{1}, N_{2}, N_{3}\right)$ выпуклое. Кроме множества неотрицательных тригонометрических многочленов, введем класс $\sigma_{P}\left(2 N_{1}, 2 N_{2}, 2 N_{3}\right)$ степенных неотрицательных многочленов вида

$$
f(x, y, z)=\sum_{0 \leqslant k \leqslant 2 N_{1}} \sum_{0 \leqslant \ell \leqslant 2 N_{2}} \sum_{0 \leqslant m \leqslant 2 N_{3}} a_{k, \ell, m} x^{k} y^{\ell} z^{m} .
$$

